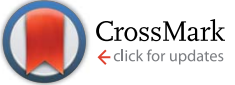

Cite this: RSC Adv., 2017, 7, 8186

\title{
Neutral plane control by using polymer/graphene flake composites for flexible displays $\uparrow$
}

\begin{abstract}
B. Hwang, $t^{a b}$ S. Lim, $t^{a}$ M. Park ${ }^{a}$ and S. M. Han*a
Controlling the neutral plane of a multilayer structure is crucial in designing a reliable flexible display, where the bending strain imposed on the individual layers that are positioned closer to the neutral plane can be significantly reduced. In this study, we explore the usage of polymer/graphene composites with different concentrations of graphene flakes to systematically vary the modulus in attempt to control the location of the neutral plane to the desirable position. Nanoindentation of the PMMA/graphene composite film on Si substrate revealed that increasing the graphene flake content led to the enhancement in hardness and modulus of the composite. The increased Young's modulus caused a shift in the neutral plane position towards the organic emissive layers by $\sim 2.2 \mu \mathrm{m}$ that resulted in a reduction of bending strain in the organic light emitting diodes with a typical multilayer structure. Furthermore, gas permeability against $\mathrm{O}_{2}$ and water molecules showed that the gas transmission rate of PMMA/graphene composites decreased as the content of graphene flakes increased since the randomly mixed graphene in PMMA can efficiently hinder the $\mathrm{O}_{2}$ and water molecules from transporting through the thickness of the composite, thereby enhancing the barrier properties.
\end{abstract}

Received 4th November 2016 Accepted 13th January 2017

DOI: 10.1039/c6ra26312g

www.rsc.org/advances typically have a thick encapsulation layer as well as a thick polymeric substrate, and thus selecting polymer materials with a desirable modulus for the substrate or the encapsulation layer is crucial in controlling the neutral plane of the multilayered structure.

Graphene is known to have extreme mechanical properties with high modulus and strength of $1 \mathrm{TPa}$ and $130 \mathrm{GPa}$, respectively. ${ }^{9-13}$ In addition, the 2-D structure of graphene can efficiently block the gas molecules from penetrating the graphene, thereby resulting in low permeation level of water or $\mathrm{O}_{2}$ molecules. ${ }^{\mathbf{1 4 , 1 5}}$ Therefore, graphene has the potential as a reinforcement material not only for enhanced mechanical properties but also for excellent barrier properties. Several studies have reported that the polymer/graphene composites are able to enhance the mechanical properties of polymer. ${ }^{16-19}$ For example, Ramanathan et al. showed that the modulus and strength can increase by $80 \%$ and $20 \%$, respectively, by adding $\sim 1.0 \mathrm{wt} \%$ of graphene to poly(methyl methacrylate) (PMMA). ${ }^{17}$ Other studies indicate enhanced gas transmission rate, and the study by Tsai et al. has shown that the water transmission rate was reduced by $180 \%$ for a composite consisting of $1.0 \mathrm{wt} \%$ reduced graphene oxide (RGO) in polyimide matrix. ${ }^{18}$

While the enhancements in the mechanical and barrier properties were reported in previous studies for polymer/ graphene composites, there is still a lack of studies exploring the potential in controlling the mechanical properties by varying the graphene content in attempt to control the neutral plane while maintaining a high gas transmission rate for flexible display applications. Therefore, the purpose of this study is

\footnotetext{
${ }^{a}$ Graduate School of Energy Environment Water and Sustainability, Korea Advanced Institute of Science \& Technology, Daejeon, 305701, Republic of Korea. E-mail: smhan01@kaist.ac.kr

${ }^{b}$ BASF Electronic Materials R\&D Center Asia, Suwon, 16419, Republic of Korea

$\dagger$ Electronic supplementary information (ESI) available. See DOI 10.1039/c6ra26312g

$\ddagger$ These authors contributed equally to the research.
} 
to investigate the effect of graphene inclusion on the modulus of polymer/graphene composite, which is then correlated with the position of neutral plane. In the present study, graphene flakes were blended in PMMA matrix for improving barrier and mechanical properties of polymer/graphene composites, and different contents of graphene flakes were evaluated for optimization. To confirm the enhancement of the modulus of polymer/graphene composites, nanoindentation was performed on each specimen. Based on the experimentally determined modulus, the shift in the neutral plane position was calculated for a typical multilayer stack used for organic light emitting diodes (OLEDs). Lastly, the gas transmission rate of PMMA/graphene with varying graphene content was also measured by using gas permeability tester to confirm the enhancement of barrier properties by the addition of graphene flake to polymer material.

\section{Experimental}

\subsection{Fabrication of PMMA/graphene flake composites}

Commercial pristine monolayer graphene flake suspension solution (Graphene Supermarket) was used in this study. The concentration of graphene flakes in the solution was a $1 \mathrm{mg} \mathrm{L}^{-1}$, and an average size of the graphene flakes was $\sim 550 \mathrm{~nm}$. The XPS result showed that the graphene flakes are highly pure with an oxygen content of $\sim 7.0 \%$ (Fig. S4a $\dagger$ ). The solution was then sonicated for $30 \mathrm{~min}$ at room temperature to enhance the dispersion during the mixing process with polymer materials. The graphene flake solution was then mixed with PMMA (Mircochem, 950000 molecular weight $11 \%$ in anisole) to fabricate the PMMA/graphene composites. To enhance the dispersion in PMMA, the graphene solution in ethanol was transferred to dimethylformamide (DMF, Sigma Aldrich, anhydrous, 99.8\%) by using filtration with a pore size of $200 \mathrm{~nm}$. The exchanged graphene in DMF solution was then blended in PMMA solution in varying graphene contents of $0,0.2,0.4,0.6,0.8$ and $1.0 \mathrm{wt} \%$. Sonication was conducted to homogeneously disperse the graphene flakes in PMMA, which resulted in dark color as shown in ESI 01.†

The fabricated PMMA/graphene solution was spin-coated on Si substrate for nanoindentation, polycarbonate (PC) for gas permeation tests, and on glass substrate for optical transparency measurements. The spin-coating time was fixed at 30 seconds and the rpm was varied between 2000-3000 rpm. Different contents of graphene in PMMA caused different coating rate, and thus appropriate rpm was selected to obtain the same thickness of PMMA/graphene composite. Thickness of $10 \mu \mathrm{m}$ was used for nanoindentation tests on Si substrate to minimize substrate effects, and $3 \mu \mathrm{m}$ for gas permeation tests on PC substrate as well as for the UV-vis transmittance measurements. The spin-coated PMMA/graphene composites were heated at $\sim 80{ }^{\circ} \mathrm{C}$ for 10 minutes in ambient air by using hot plate. Thicknesses of the fabricated PMMA/graphene composites were confirmed using atomic force microscopy (AFM, JPK, NanoWizard ${ }^{\circledR} 3$ NanoScience). The transparency of the PMMA/graphene composite was measured using the UV-vis spectrometer (Micro-Light, MT-500) in the range of 350-
$800 \mathrm{~nm}$. The optical microscope images of the PMMA/graphene composites confirmed that the graphene flakes were homogeneously distributed in PMMA, as shown in ESI 02. $\dagger$ The average sizes of graphene flakes in PMMA/graphene composites were $\approx 1.1 \mu \mathrm{m}$ for the PMMA with $0.2 \mathrm{wt} \%$ graphene, which was increased to $\approx 2.9 \mu \mathrm{m}$ as the graphene flake contents increased. High contents of graphene in PMMA led to the agglomerations of graphene flakes, thereby resulting in the increase in average size of graphene flakes in PMMA.

\subsection{Nanoindentation}

Mechanical properties of PMMA/graphene composites were characterized using nanoindentation (MTS, Nano Indenter XP) with a Berkovich tip. Continuous stiffness measurement (CSM) method was used in a load-controlled mode with a maximum load of $100 \mathrm{mN}$. 16 positions were indented for each sample, and the average values of those results are presented. The hardness and Young's modulus presented throughout this paper were calculated by using Oliver and Pharr (O\&P) model, which is explained in detail in ref. 20. The thin polymer film on a stiffer Si substrate can cause error in the calculation of contact areas during indentation due to the pile-up of the softer film material especially in the shallow displacement region, and this can cause large errors in using pre-calibrated O\&P area function. ${ }^{21-23}$ The Han-Yu-Vlassak (HYV) model can be used to calculate the contact area based on the contact stiffness, thereby correcting the error associated with inaccurate contact areas. ${ }^{21,23}$ Therefore, the hardness was also calculated by using HYV model for comparison to that of the O\&P model.

\subsection{Gas permeability test}

$\mathrm{O}_{2}$ permeation tester (OX-TRAN 2/21 MD (MOCON)) and water permeation tester (PERMATRAN_W 3/33 MA (MOCON)) were used to evaluate the $\mathrm{O}_{2}$ and water transmission rate of the PMMA/graphene composites on PC substrate, respectively. The permeation tests were conducted at room temperature by following a standard testing method for vapor transmission rate through plastic films and sheets using a modulated infrared sensor (ASTM F1249 for $\mathrm{O}_{2}$ and ASTM D3985 for water).

\section{Results and discussion}

\subsection{Nanoindentation on PMMA/graphene flake composites}

Young's modulus and hardness analyzed using the O\&P method for the PMMA/graphene composites as a function of graphene flake concentration ranging from $0 \mathrm{wt} \%$ to $1.0 \mathrm{wt} \%$ are shown in Fig. 1. The modulus for the PMMA without graphene increased with increase in displacement, as expected for soft, compliant polymer thin film (10.0 $\mu \mathrm{m}$ PMMA) on hard, stiff Si substrate. The modulus was therefore extrapolated from the shallow displacement range after fine tuning the tip area calibration in that range as explained in the work by Han et al. ${ }^{23}$ to minimize the substrate effect. Similarly, the modulus for samples containing graphene flakes with concentration in the range of 0.2 to $1.0 \mathrm{wt} \%$ were analyzed to extrapolate the Young's modulus from the O\&P method. The extrapolated modulus for the PMMA 

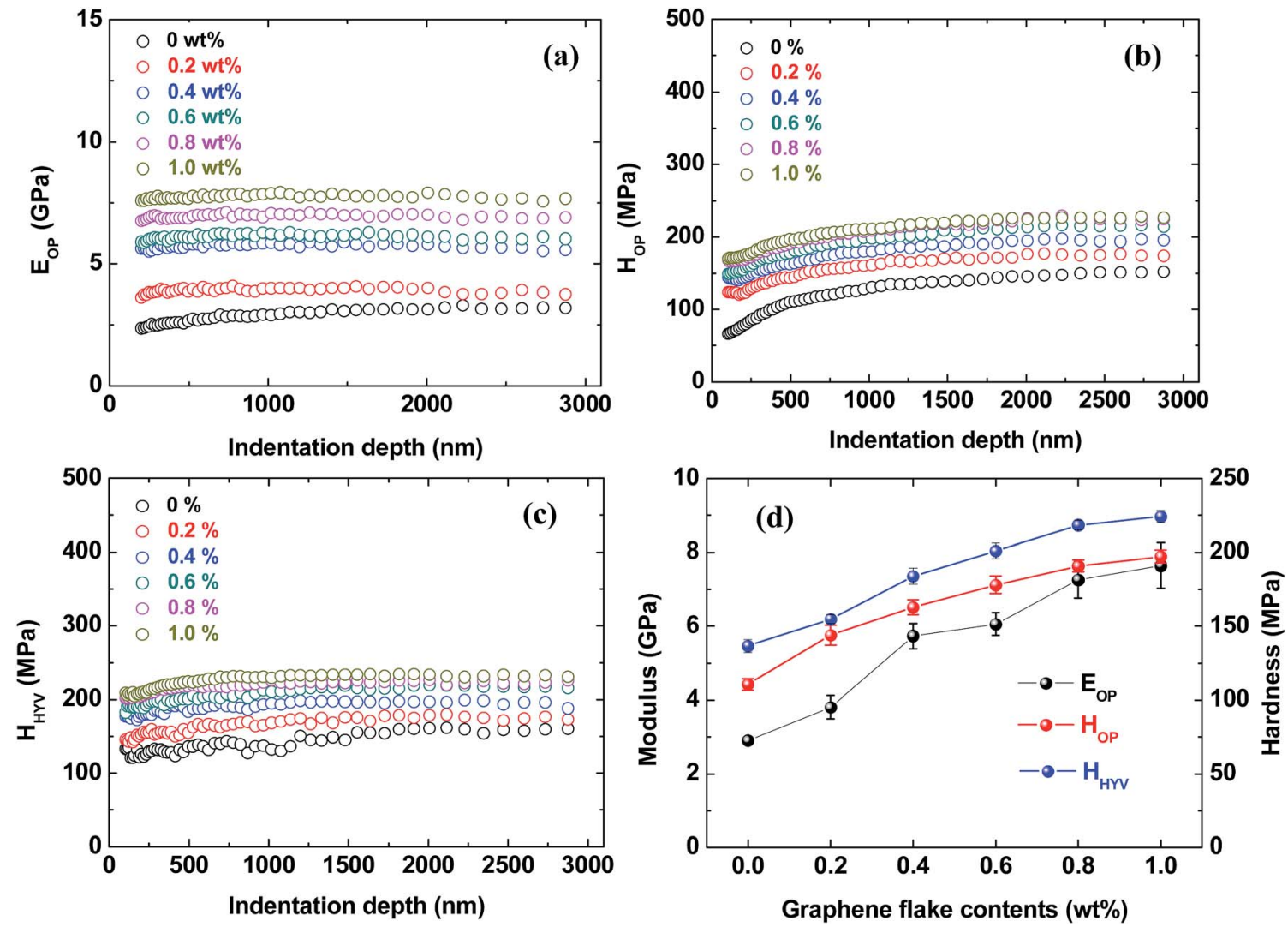

Fig. 1 Nanoindentation results as a function of the contents of graphene flake in PMMA. (a) Young's modulus vs. indentation displacement, (b) hardness (O\&P) vs. indentation displacement, (c) hardness (HYV) vs. indentation displacement and (d) extracted modulus and hardness values at $500 \mathrm{~nm}$ as a function of graphene content in PMMA.

without graphene showed $2.9 \mathrm{GPa}$ that increased to $7.7 \mathrm{GPa}$ for the PMMA with $1.0 \mathrm{wt} \%$ of graphene flakes.

Hardness also increased as the contents of graphene flakes increased as shown in Fig. 1b, in which the PMMA without graphene flake showed a O\&P hardness $\left(H_{\mathrm{OP}}\right)$ of $110 \mathrm{MPa}$ while the $H_{\mathrm{OP}}$ of $197 \mathrm{MPa}$ at $500 \mathrm{~nm}$ was obtained for the PMMA with $1.0 \mathrm{wt} \%$ of graphene flakes. For comparison, the hardness $\left(H_{\mathrm{HYV}}\right)$ was also calculated by the aforementioned HYV model, in which the contact areas are determined based on the measured stiffness (Fig. 1c). The experimentally determined O\&P modulus of PMMA/graphene and the known elastic constants of Si substrate as well as the thickness of the film were used as input to calculate $H_{\mathrm{HYV}}$. $H_{\mathrm{HYV}}$ showed similar trend of increasing hardness with an increase in the graphene flake content, where the PMMA with $1.0 \mathrm{wt} \%$ graphene and without graphene showed the $H_{\mathrm{HYV}}$ of $224 \mathrm{MPa}$ and $137 \mathrm{MPa}$ at $500 \mathrm{~nm}$, respectively. Since $H_{\mathrm{HYV}}$ of PMMA/graphene composites showed a plateau in shallow indentation depth, $H_{\mathrm{HYV}}$ can be a more accurate measure of the hardness than the $H_{\mathrm{OP}}$ for this case of polymer on stiff substrate system. Hardness were extracted at the displacement of $500 \mathrm{~nm}$, which is $5 \%$ of the thickness of PMMA/graphene composite film, are plotted against the graphene content to clearly show the trend of increasing hardness with increasing graphene content (Fig. 1d). The reinforcement ability of graphene flakes in PMMA was better than other types of graphene, such as reduced graphene oxide (RGO) or graphene oxide (GO), which was attributed to the larger defect density of RGO or GO layers than that of graphene flakes (ESI 03 and $04 \dagger$ ).

Based on the volume fraction of graphene flakes in PMMA, the predicted Young's modulus and hardness are stated in Table 1 along with the experimentally measured values. Here, the Young's modulus and hardness for a single graphene flake was taken to be $0.92 \mathrm{TPa}$ and $10 \mathrm{GPa}$, respectively from ref. 24 and 25 . The modulus determined by nanoindentation revealed that modulus of PMMA/graphene composites linearly increased as the contents of graphene flakes increased, as shown in Fig. 1d. Furthermore, it should be noted that the analytically estimated modulus and hardness of PMMA/graphene composites showed similar values and tendency with those of experimentally determined values. The consistency of experimentally and analytically determined modulus and hardness confirmed the effectiveness of graphene flakes uniformly distributed in the PMMA as the strength enhancer. Since the analytical calculation used the modulus and hardness value of 2-D graphene sheet measured under in-plane tensile straining condition, ${ }^{24,25}$ the anisotropic graphene flakes distributed in PMMA with randomly varying orientations might be the cause for the small discrepancies in modulus and hardness values between the experimentally and analytically determined values. 
Table 1 Experimentally and analytically determined modulus and hardness values as a function of graphene flake content

\begin{tabular}{|c|c|c|c|c|c|}
\hline $\begin{array}{l}\text { Graphene flake } \\
\text { content (wt\%) }\end{array}$ & $E_{\mathrm{OP}}(\mathrm{GPa})$ & $E(\mathrm{GPa})$ (calculation) & $H_{\mathrm{OP}}(\mathrm{MPa})$ & $H_{\mathrm{HYV}}(\mathrm{MPa})$ & $H(\mathrm{MPa})$ (calculation) \\
\hline 0 & 2.9 & 3.1 & 110.5 & 136.6 & 187.0 \\
\hline 0.4 & 5.7 & 6.3 & 162.9 & 183.9 & 194.0 \\
\hline 0.6 & 6.1 & 7.9 & 178.1 & 201.1 & 198.7 \\
\hline 0.8 & 7.3 & 9.4 & 190.8 & 218.4 & 202.5 \\
\hline
\end{tabular}

The reported modulus variance with the inclusion of graphene flakes is expected to be useful in designing a reliable flexible device where the neutral plane can be shifted to the desirable position by varying the modulus of the encapsulation layer. The neutral plane is given by $\varepsilon=y / R$, where $y$ and $R$ are the distance from neutral plane and the radius of bending curvature, respectively. Therefore, modulus control of encapsulation layer can be a simple yet effective methodology for controlling the neutral plane in a flexible device with extremely thick layer of encapsulation typically in the range of $\sim 1.3 \mu \mathrm{m}$ (Fig. S5 $\dagger$ ). For an organic light emitting diodes (OLEDs) that consist of multilayers, the organic emissive layer is the most sensitive part when subjected to bending strain. To minimize the damage on the organic emissive layer under bending, the neutral plane should therefore be located near the emissive layer. By assuming a typical structure of flexible OLEDs as shown in Fig. S6, $\uparrow$ the use of PMMA/graphene composites encapsulation layer can shift the neutral plane to the desired position (here, to organic emissive layer) due to the increased modulus as a result of graphene addition. If PMMA with $1.0 \mathrm{wt} \%$ of graphene flakes is used for encapsulation, a shift in the neutral plane to the position to $105.84 \mu \mathrm{m}$ from $103.64 \mu \mathrm{m}$ is expected. For a hypothetical bending radius of $10 \mathrm{~mm}$, the shift in the neutral plane of $2.2 \mu \mathrm{m}$ is expected that can decrease the applied strain to the organic layers from $5.9 \%$ to $5.0 \%$ as determined by $\varepsilon=y / R$. In other words, the increase in the modulus by mixing the graphene flakes with PMMA can result in the significant reduction in the applied strain to the organic layers, thereby resulting in enhancing the performance and reliability of the device under cyclic bendings.

\subsection{Gas permeability of PMMA/graphene flake composites}

$\mathrm{O}_{2}$ transmission rate (OTR) and water vapor transmission rate (WVTR) of PMMA/graphene composites were measured by using gas permeability tester to investigate the effect of graphene flake contents on the gas transmission rate. The PMMA/ graphene composites with thickness of $3 \mu \mathrm{m}$ on PC substrates were prepared to have different contents of graphene flakes in PMMA ranging from 0 to $1.0 \mathrm{wt} \%$ for the gas permeability tests. Since gas transmission rates of polycarbonate are significantly higher than those of PMMA, the PC substrates were selected to minimize the substrate effects on the gas permeability results for the PMMA/graphene composites. The results of OTR and WVTR of PMMA/graphene composites as a function of the graphene flake concentration are presented in Fig. 2. The
PMMA without graphene showed OTR value of $769 \mathrm{~g}$ per $\mathrm{m}^{2}$ per day that decreased to $45 \mathrm{~g}$ per $\mathrm{m}^{2}$ per day as the contents of graphene flakes increased to $1.0 \mathrm{wt} \%$, as shown in Fig. 2. The lowest OTR value was achieved in the specimens with $1.0 \mathrm{wt} \%$ of graphene flakes in PMMA, which showed $~ 90 \%$ lower value compared to that of PMMA without graphene. The result of WVTR showed a similar behavior with that of OTR where the WVTR values decreased from $55.4 \mathrm{~g}$ per $\mathrm{m}^{2}$ per day to $12.6 \mathrm{~g}$ per $\mathrm{m}^{2}$ per day as contents of graphene flakes in PMMA increased from $0 \mathrm{wt} \%$ to $1.0 \mathrm{wt} \%$. $\sim 80 \%$ decrease in WVTR value was achieved in the PMMA with $1.0 \mathrm{wt} \%$ graphene flakes.

The enhancement of barrier properties of PMMA with the addition of graphene flakes is attributed to the blocking the gas or water molecule transmission through PMMA by the graphene. In the PMMA without graphene, gas or water molecules are able to transit through PMMA without significant disturbance, which leads to the high OTR and WVTR (Fig. S7a $\dagger$ ). Since graphene flakes have a low permeability to gas or water molecules, however, the paths for the gas transmission through PMMA are efficiently blocked by graphene flakes after the distribution of graphene flakes in PMMA (Fig. S7b†). The high contents of graphene will cause the increased probability of the blocking gas or water transmission through PMMA, and thus, the lowest values of OTR and WVTR were observed in the maximum content of graphene flakes, $1.0 \mathrm{wt} \%$.

Although both OTR and WVTR were significantly higher in the PMMA/graphene composite than in some of the standard

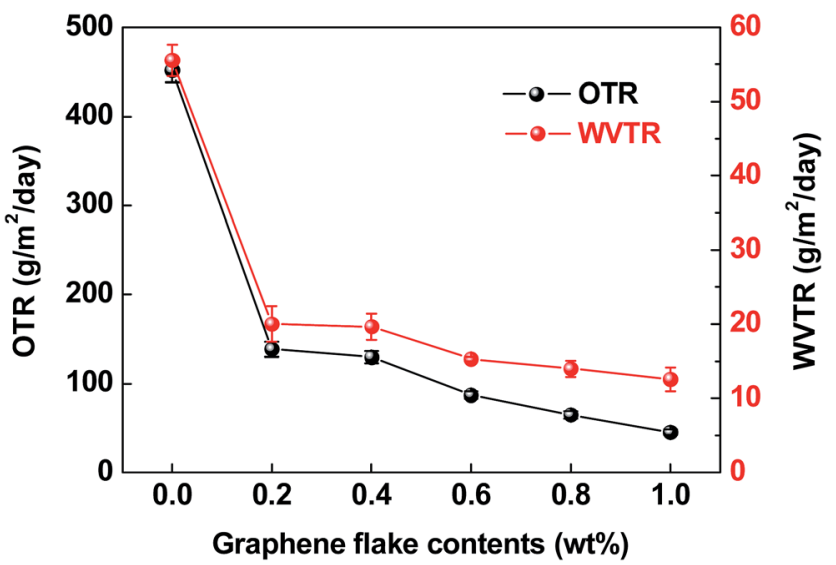

Fig. 2 Oxygen transmission rate and water vapor transmission rate of PMMA with different contents of graphene flake. 
encapsulation materials for OLEDs such as the $\mathrm{SiN}_{x}, \mathrm{Al}_{2} \mathrm{O}_{3}, \mathrm{ZrO}_{2}$ or $\mathrm{SiO}_{2}$ (ref. 26-28) due to the limited barrier properties of PMMA, the addition of graphene flake into PMMA clearly showed remarkably reduction in the OTR and WVTR. With further development of other polymer/graphene composites, the gas transmission rate has a great potential for enhancement. In comparison to the inorganic barriers such as $\operatorname{SiN}_{x}$, $\mathrm{Al}_{2} \mathrm{O}_{3}, \mathrm{ZrO}_{2}$ or $\mathrm{SiO}_{2},{ }^{26-28}$ the polymer/graphene composites are expected to be cost effective in terms of material cost and processing as well as allowing for the needed mechanical flexibility that would be well suited for the flexible display application.

\subsection{Optical transmittance of PMMA/graphene flake composites}

Optical transmittance was measured for the PMMA with varying concentration of graphene flakes to confirm the transparency of the PMMA/graphene composites. The PMMA/graphene composites with the thickness of $3 \mu \mathrm{m}$ were deposited on glass substrates for the transmittance measurement. Fig. 3 shows the optical transmittance across the visible wave length spectrum for PMMA with different wt $\%$ of graphene flakes. The total transmittance decreased as the contents of graphene flakes increased, as expected. The optical transmittance at a wave length of $550 \mathrm{~nm}$ was $94 \%$ for the PMMA with $1.0 \mathrm{wt} \%$ of graphene flakes while $99 \%$ transmittance was observed for the PMMA without graphene. A mono layer graphene is known to decrease the optical transmittance by $\sim 2.7 \%,{ }^{29}$ and thus, an increase in the graphene flake concentration in PMMA is expected to decrease the optical SSS transmittance as experimentally observed. However, the lowest value of optical transmittance of present study, i.e. the result of the PMMA/graphene composites with $1.0 \mathrm{wt} \%$ of graphene flakes, was highly transparent (Fig. $\mathrm{S} 8 \dagger$ ), and still above the requirement for the optical transmittance of encapsulation materials for OLEDs, $\sim 90 \%{ }^{30}$ Therefore, the loss of optical properties will not limit the application of graphene flakes for the encapsulation material for flexible displays.

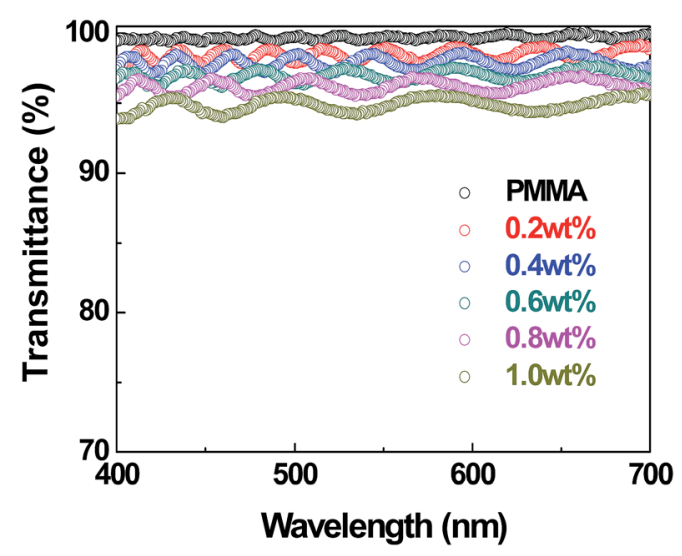

Fig. 3 Optical transmittance of PMMA with different contents of graphene flake as a function of wavelength.

\section{Conclusion}

In this study, the effect of varying the graphene flake content in the mechanical properties of PMMA/graphene composite was explored with the focus of controlling the neutral plane for strain engineering of a flexible display by potentially replacing the encapsulation layer with the proposed PMMA/graphene composite. Excellent mechanical properties of graphene resulted in the increase in hardness from $137 \mathrm{MPa}$ to $224 \mathrm{MPa}$ and Young's modulus from 2.9 GPa to $7.7 \mathrm{GPa}$ for the PMMA/ graphene composite as the graphene content increased from 0 to $1.0 \mathrm{wt} \%$. The calculation of neutral plane based on the measured mechanical properties of PMMA/graphene composites indicated that the neutral plane in OLEDs with the PMMA/ graphene composites used as the encapsulation layer can be shifted towards the organic layers; $1.0 \mathrm{wt} \%$ graphene flake inclusion with modulus of $7.7 \mathrm{GPa}$ results in a shift in the neutral plane by $2.2 \mu \mathrm{m}$ that translates to a reduction in the bending strain in the organic emissive layer from 5.9\% to 5.0\% for a typical OLED multilayer structure. Furthermore, PMMA/ graphene composites showed lower $\mathrm{O}_{2}$ and water molecule gas transmission rates compared to those of PMMA without graphene as the randomly distributed graphene flakes in the PMMA act as an efficient barrier. Our findings indicate that the polymer/graphene composites can enhance not only the barrier properties, but also contribute to the enhancement in the reliability of flexible displays by controlling the neutral plane of the multilayer structure such that the strain imposed on the emissive organic layer is minimized.

\section{Acknowledgements}

The authors would like to acknowledge the financial support from the National Research Foundation of Korea (NRF) grant funded by the Korea government (MSIP) (No. NRF2016R1A2B3011473) and the Center for Advanced MetaMaterials (CAMM) funded by the Ministry of Science, ICT and Future Planning as Global Frontier Project (CAMM-No. 2014063701, 2014063700).

\section{Notes and references}

1 D.-U. Jin, J.-S. Lee, T.-W. Kim, S.-G. An, D. Straykhilev, Y.-S. Pyo, H.-S. Kim, D.-B. Lee, Y.-G. Mo, H.-D. Kim and H.-K. Chung, SID Int. Symp. Dig. Tech. Pap., 2009, 40, 983985.

2 M. D. J. Auch, O. K. Soo, G. Ewald and C. Soo-Jin, Thin Solid Films, 2002, 417, 47-50.

3 M. Katsuhara, I. Yagi, M. Noda, N. Hirai, R. Yasuda, T. Moriwaki, S. Ushikura, A. Imaoka, K. Nomoto, A. Yumoto and T. Urabe, SID Int. Symp. Dig. Tech. Pap., 2009, 40, 656-659.

4 L. Hu, H. Wu and Y. Cui, MRS Bull., 2011, 36, 760-765.

5 J. Lewis, Mater. Today, 2006, 9, 38-45.

6 C.-J. Chiang, C. Winscom, S. Bull and A. Monkman, Org. Electron., 2009, 10, 1268-1274. 
7 B. Hwang, T. Kim and S. M. Han, Extreme Mechanics Letters, 2016, 8, 266-272.

8 B. Hwang, H.-A. S. Shin, T. Kim, Y.-C. Joo and S. M. Han, Small, 2014, 10, 3397-3404.

9 A. K. Geim, Science, 2009, 324, 1530-1534.

10 M. Huang, T. A. Pascal, H. Kim, W. A. Goddard and J. R. Greer, Nano Lett., 2011, 11, 1241-1246.

11 Y. Kim, J. Lee, M. S. Yeom, J. W. Shin, H. Kim, Y. Cui, J. W. Kysar, J. Hone, Y. Jung, S. Jeon and S. M. Han, Nat. Commun., 2013, 4, 2114.

12 C. Lee, X. Wei, J. W. Kysar and J. Hone, Science, 2008, 321, 385-388.

13 Y. Zhu, S. Murali, W. Cai, X. Li, J. W. Suk, J. R. Potts and R. S. Ruoff, Adv. Mater., 2010, 22, 3906-3924.

14 B. Hwang, M. Park, T. Kim and S. M. Han, RSC Adv., 2016, 6, 67389-67395.

15 H. W. Kim, H. W. Yoon, S.-M. Yoon, B. M. Yoo, B. K. Ahn, Y. H. Cho, H. J. Shin, H. Yang, U. Paik, S. Kwon, J.-Y. Choi and H. B. Park, Science, 2013, 342, 91-95.

16 H. Kim, A. A. Abdala and C. W. Macosko, Macromolecules, 2010, 43, 6515-6530.

17 T. Ramanathan, A. A. Abdala, S. Stankovich, D. A. Dikin, M. Herrera Alonso, R. D. Piner, D. H. Adamson, H. C. Schniepp, X. Chen, R. S. Ruoff, S. T. Nguyen, I. A. Aksay, R. K. Prud'Homme and L. C. Brinson, Nat. Nanotechnol., 2008, 3, 327-331.
18 M.-H. Tsai, I. H. Tseng, Y.-F. Liao and J.-C. Chiang, Polym. Int., 2013, 62, 1302-1309.

19 D. Barun, K. E. Prasad, U. Ramamurty and C. N. R. Rao, Nanotechnology, 2009, 20, 125705.

20 W. C. Oliver and G. M. Pharr, J. Mater. Res., 1992, 7, 15641583.

21 S. M. Han, R. Saha and W. D. Nix, Acta Mater., 2006, 54, 1571-1581.

22 R. Saha and W. D. Nix, Acta Mater., 2002, 50, 23-38.

23 S. M. Han, E. P. Guyer and W. D. Nix, Thin Solid Films, 2011, 519, 3221-3224.

24 S. Bera, A. Arnold, F. Evers, R. Narayanan and P. Wölfle, Phys. Rev. B: Condens. Matter Mater. Phys., 2010, 82, 195445.

25 M. Poot and H. S. J. van der Zant, Appl. Phys. Lett., 2008, 92, 063111.

26 D. Yu, Y.-Q. Yang, Z. Chen, Y. Tao and Y.-F. Liu, Opt. Commun., 2016, 362, 43-49.

27 Y.-H. Choi, Y. G. Lee, X. Bulliard, K.-H. Lee, S. Lee, D. Choi, J.-J. Park and J. M. Kim, Scr. Mater., 2010, 62, 447-450.

28 W. Huang, X. Wang, M. Sheng, L. Xu, F. Stubhan, L. Luo, T. Feng, X. Wang, F. Zhang and S. Zou, Mater. Sci. Eng., B, 2003, 98, 248-254.

29 X. Li, Y. Zhu, W. Cai, M. Borysiak, B. Han, D. Chen, R. D. Piner, L. Colombo and R. S. Ruoff, Nano Lett., 2009, 9, 4359-4363.

30 A. P. Ghosh, L. J. Gerenser, C. M. Jarman and J. E. Fornalik, Appl. Phys. Lett., 2005, 86, 223503. 